Palabras clave: Quejas de memoria, Depresión, Cognición.

\title{
Una disminución de las quejas de memoria se asocia con mejoría en el estado de ánimo: un estudio de seguimiento a los doce meses en pacientes deprimidos
}

\author{
Risto Antikainen* \\ Kirsi Honkalampi ${ }^{\star}$ \\ Tuomo Hänninen** \\ Heli Koivumaa-Honkanen* \\ Antti Tanskanen* \\ Kaisa Haatainen* \\ Jukka Hintikka* \\ Heimo Viinamäki ${ }^{\star}$ \\ * Department of Psychiatry, Kuopio University \\ Hospital \\ ** Department of Neurology, Kuopio University \\ Hospital
}

FINLAND

\begin{abstract}
RESUMEN - El objetivo de nuestro estudio de seguimiento a los doce meses fue investigar las quejas de memoria en pacientes adultos, clínicamente deprimidos, neurológicamente sanos, centrándose en la relación entre las quejas del estado de ánimo y de memoria. El trastorno de memoria subjetivo fue evaluado utilizando el Memory Complaint Questionnaire (MCQ) en una muestra de 174 pacientes adultos (edad media 44 años , rango 21-64 años) con depresión. Los niveles de función cognoscitiva, incluyendo la memoria, se evaluaron utilizando una batería de tests neuropsicológicos. Las características del ánimo y de la personalidad fueron evaluadas mediante escalas, que incluyeron el Beck Depression Inventory (BDI) y la Hamilton Depression Rating Scale (HDRS). Se repitieron todas las mediciones en el seguimiento después de seis y doce meses. Los cambios en las quejas de memoria durante el período de seguimiento se asociaron sobre todo con cambios en el estado de ánimo, síntomas mentales, características alexitímicas y capacidad psicosocial, pero no con el funcionamiento cognoscitivo. Concluímos que la mejoría de la depresión en los pacientes adultos, neurológicamente sanos, normalmente se traduce en una mejoría del deterioro subjetivo de la memoria. Este hallazgo podría emplearse para motivar a un paciente deprimido con deterioro subjetivo de memoria a buscar tratamiento.
\end{abstract}




\section{Introducción}

Los trastornos de memoria subjetivos o las quejas de memoria son bastante comunes en la edad adulta, incluyendo adultos de mediana edad, e incluso adultos jóvenes. En un gran estudio por correo se documentó una alta prevalencia de falta de memoria percibida subjetivamente en sujetos holandeses sanos jóvenes (29\%) y de mediana edad (34\%) (Commissaris, Ponds y Jolles 1998). La necesidad de diferenciar entre los distintos tipos de quejas de memoria (Derouesne y Lacomblez 2002) y entre grupos de edad está clara.

Las quejas de memoria han sido estudiadas mayoritariamente en el contexto del deterioro de memoria asociado a la edad (Crook, Feher y Larrabee 1992), con resultados más bien contradictorios. En una revisión de estudios recientes, Jonker, Geerlings y Schmand (2000) concluyeron que las quejas de memoria simplemente no deberían considerarse como un fenómeno relacionado con la edad o como un síntoma de depresión, sino como un posible signo prodrómico de demencia. Esta visión fue apoyada por Clarnette et al. (2001) y Stewart et al. (2001). Sin embargo, sobre la base de un estudio longitudinal en una muestra de población de la comunidad, de 70 años de edad o mayores, Jorm et al. (2001) documentaron que, aunque las quejas de memoria reflejan percepciones pasadas del funcionamiento de la memoria, y aunque puedan ser también una manifestación temprana de deterioro de la memoria, los afectos negativos actuales (ansiedad y depresión) están asociados con las quejas de memoria. En línea con estos resultados, Comijs et al. (2002) encontraron en un estudio de seguimiento a los 6 años que en las personas más mayores, sin mostrar declive cognoscitivo real, las quejas de problemas de memoria podrían reflejar problemas psicoafectivos y de salud.

En la práctica clínica, los pacientes adultos que se quejan de deterioro de la memoria pero que no muestran síntomas neurológicos también son bastante comunes. Con bastante frecuencia, el deterioro subjetivo despierta temor y ansiedad (Commissaris, Ponds y Jolles 1998). Sin embargo, en este grupo de edad no hay acuerdo general en la etiología o en la significación clínica del deterioro de la memoria. Chandler y Gerndt (1988) documentaron que las quejas de memoria eran un marcador para la depresión en población geriátrica (más de 60 años) pero no en pacientes psiquiátricos ingresados más jóvenes. McDonald, Cope y David (1993) encontraron que en aquéllos que acudían a medicina general, el deterioro cognoscitivo subjetivo estaba fuertemente relacionado con el trastorno psiquiátrico, especialmente con un estado de ánimo deprimido, pero no con la fatiga, ansiedad, o funcionamiento objetivo. En sujetos cognoscitivamente normales divididos en dos grupos de edad, por debajo y por encima de los 50 años, Derouesne et al. (1999) no encontró relación próxima entre la gravedad de las quejas de memoria y el funcionamiento de la memoria. Las quejas de memoria en ambos grupos de edad estaban fuertemente relacionadas con el estado afectivo, principalmente con la gravedad de la ansiedad. En una muestra de pacientes adultos, con diagnósticos heterogéneos de derivación, incluyendo daño cerebral y enfermedades neurológicas, Rohling et al. (2002) encontraron que los pacientes con depresión refieren más quejas de memoria. No se encontró asociación entre el estado de ánimo y el funcionamiento neurocognoscitivo objetivo.

Se ha encontrado frecuentemente deterioro cognoscitivo leve en casos de depresión y se asume que mejore en la remisión. Un estudio de seguimiento reciente en pacien- 
tes que padecían depresión y en sujetos controles emparejados confirmó el deterioro del funcionamiento cognoscitivo, pero no mostró normalización del funcionamiento en un retest tras la remisión de la depresión (Reischies y Neu 2000).

En nuestro anterior estudio de seguimiento a los seis meses (Antikainen et al. 2001) se investigaron asociaciones entre el funcionamiento cognoscitivo, gravedad de la depresión y de las quejas de memoria en pacientes adultos deprimidos, neurológicamente sanos, al inicio del tratamiento ambulatorio, y seis meses más tarde. En contraste con estudios previos, nuestro criterio de inclusión para un diseño longitudinal fue depresión y el centro de atención, la relación entre el estado de ánimo y las quejas de memoria. Los resultados sugirieron que, normalmente, los problemas subjetivos de memoria disminuyen si se mejora la depresión. Sin embargo, hallazgos inconsistentes de estudios previos han indicado la necesidad de un período de seguimiento mayor. Por tanto, continuamos la investigación en nuestra muestra previa, con un seguimiento adicional a los doce meses desde la evaluación inicial. El objetivo fue investigar la estabilidad de las asociaciones entre las quejas de memoria, el funcionamiento cognoscitivo y el estado de ánimo en una muestra de pacientes adultos clínicamente deprimidos y neurológicamente sanos. Nos propusimos, también como objetivo, identificar factores asociados con el cambio en las quejas de memoria.

\section{Método}

\section{Muestra y procedimiento}

La muestra inicial fueron 203 pacientes consecutivos (84 hombres, 119 mujeres), de edades comprendidas entre los 21 y los 64 años, y con sospecha de depresión, que habían sido remitidos para tratamiento psiquiátrico ambulatorio al Departamento de Psiquiatría del Kuopio University Hospital, en Kuopio, Finlandia. Se consideró que un paciente reunía los criterios de inclusión para este estudio si se le diagnosticaba clínicamente depresión con criterios CIE-10 (WHO 1992) (F31.3 - 5; F32 - F34, F41.2). Los pacientes firmaron un consentimiento informado para participar en el estudio. Se excluyeron los pacientes con enfermedades del sistema nervioso central, otras enfermedades graves (infarto de miocardio reciente, ACV reciente, etc.), adictos a drogas o alcohol, aquéllos con deterioro cognoscitivo grave, o aquéllos con otros trastornos mentales graves, tales como la esquizofrenia $\mathrm{u}$ otras psicosis.

Diez pacientes (5\%) se negaron a participar desde el principio y cinco pacientes (3\%) se excluyeron por enfermedades del sistema nervioso central (por ejemplo, enfermedad de Alzheimer). En el seguimiento seis meses después, otros 19 pacientes se negaron a continuar participando, y en el seguimiento doce meses después se negaron otros 8 pacientes. La muestra final consistió, por tanto, en 161 pacientes (tasa de pérdida: $21 \%$ ). Además, algunos participantes rechazaron los tests psicológicos. En total, 174 pacientes participaron en los tests psicológicos en la evaluación inicial, 156 en el seguimiento a los seis meses y 145 en el seguimiento a los doce meses. En otra publicación se presenta información más detallada sobre la muestra y la recogida de datos (Antikainen et al. 2001).

En la evaluación inicial, el diagnóstico de trastorno depresivo se confirmó por medio de la entrevista clínica estructurada Structured Clinical Interview del DSM III-R (SCID I) (Spitzer et al. 1990; 1992). En la evalua- 
ción inicial, 188 pacientes fueron diagnosticados de depresión mayor $(n=137)$, de otros trastornos depresivos (por ejemplo, distimia o trastorno de adaptación, $\mathrm{n}=32$ ), y de síntomas depresivos subclínicos $(\mathrm{n}=$ 19). En este último grupo de 19 pacientes, la puntuación media en la evaluación inicial en el BDI ( \pm d.s.) en fue de 13,6 \pm 9,2 y en el HDRS fue de 10,7 $\pm 3,8$.

\section{Instrumentos}

El rendimiento cognoscitivo lo evaluó un psicólogo clínico a quien no se le informó sobre el diseño del estudio. En la evaluación inicial, todos los pacientes completaron cinco subescalas de la Wechsler Adult Intelligence Scale (WAIS-R) (Wechsler 1981), adaptada para su uso en población finlandesa. El nivel cognoscitivo verbal (CIV) se evaluó utilizando tres escalas (Semejanzas, Vocabulario,) y el nivel de funcionamiento cognoscitivo (CIM) utilizando dos escalas (Claves de números y Bloques). En los seguimientos a los seis y doce meses, todos los sujetos completaron tres escalas ("Span" de dígitos, Claves de números y Bloques).

La función de memoria se midió utilizando el Logical Memory Complaint Questionnaire (MCQ) (Crook, Feher y Larrabee 1992). En el MCQ, se pregunta a los sujetos sobre cómo funciona su memoria comparado con cuando eran jóvenes. Se pide a los sujetos que describan, utilizando una encuesta tipo Likert, su habilidad para desarrollar tareas habituales para las que es necesaria la memoria (recordar personas, cosas, números de teléfono, noticias e ítems de listas de la compra). También se pide a los sujetos que evalúen el deterioro global de su memoria. La puntuación resultante en la queja de memoria puede estar en un rango entre 7 y 35. A mayor puntuación, mayor es el deterioro subjetivo de memoria.

Otros instrumentos utilizados tanto en la evaluación inicial como en cada uno de los seguimientos fueron los siguientes: Beck Depression Inventory (BDI; Beck, Steer y Harbin 1988), Hamilton Depression Rating Scale (HDRS; Hamilton 1960), Symptom Check List (SCL-90; (Derogatis, Lipman y Covi 1973; Holi, Sammallahti y Aalberg 1998), y la Toronto Alexithymia Scale (TAS20; Bagby, Parker y Taylor 1994; Joukamaa et al. 2001). Las capacidades de funcionamiento general social y ocupacional se estimaron utilizando la escala SOFAS (Goldman, Skodol y Lave 1992). Los pacientes evaluaron su satisfacción vital (LS) utilizando una escala de cuatro ítems (puntuación posible entre 4 y 20) (Koivumaa-Honkanen et al. 1999).

\section{Análisis estadísticos}

Los cambios en el funcionamiento cognoscitivo y en el estado de ánimo se estudiaron con la t de Student para muestras emparejadas. Las asociaciones del cambio durante el seguimiento se investigaron dividiendo la muestra en dos grupos: aquéllos en los que las quejas de memoria habían disminuído y aquéllos en los que no habían cambiado o habían aumentado (otros). La significación de las diferencias entre ambos grupos se midió con la t de Student para muestras idependientes y emparejadas en relación con variables continuas. Se desarrollaron modelos de regresión logística (método de entrada directa) para identificar los factores independientes asociados con el cambio en las puntuaciones MCQ. Las asociaciones entre los cambios en depresión, funciones de memoria y quejas de memoria 
se estudiaron mediante análisis de la varianza de medidas repetidas. En estos análisis se utilizó un punto de corte del BDI de 19 puntos, que corresponde a depresión moderada o grave (Beck, Steer y Harbin 1988).

\section{Resultados}

En la evaluación inicial, la edad media de nuestra muestra fue de 44,3 $\pm 9,9$ años. Un $59 \%$ de los pacientes eran mujeres, un $64 \%$ estaban casados o con pareja, un $72 \%$ habían superado al menos los exámenes de educación secundaria (12 ó más años de educación formal), y un 53\% estaban de baja por enfermedad o jubilados. Los sujetos habían padecido un estado de ánimo depresivo desde hacía 9,8 \pm 10,3 años de acuerdo a su propia estimación. Las capacidades cognoscitivas de todos los sujetos (Wechsler 1981) correspondían a la media finlandesa (CIV 99,6 \pm 13,5; CIM 100,5 \pm 15,6).

En el seguimiento al año, se encontró que los pacientes depresivos habían mejorado de forma continua la velocidad y precisión motoras (Clave de dígitos), la percepción visual (Bloques), y la memoria lógica (recuerdos directo y diferido de una historia

Tabla I

Cambios en el rendimiento cognoscitivo y en las quejas de memoria a los 6 y 12 meses de seguimiento en pacientes adultos deprimidos ambulatorios

\begin{tabular}{|c|c|c|c|c|}
\hline $\begin{array}{c}\mathrm{Al} \\
\text { comienzo } \\
\text { del tratamiento } \\
(\mathrm{n}=174)\end{array}$ & $\begin{array}{c}\text { A los } \\
6 \\
\text { meses } \\
(n=156)\end{array}$ & g.l. & $\mathrm{p}^{1}$ & $\begin{array}{c}\text { A los } \\
12 \\
\text { meses } \\
(\mathrm{n}=145)\end{array}$ \\
\hline
\end{tabular}

Cognición general (puntuaciones, media \pm d.e.)

\begin{tabular}{|c|c|c|c|c|c|c|c|}
\hline WAIS-R, semejanzas & $13,1 \pm 2,2$ & & & & & & \\
\hline WAIS-R, vocabulario & $22,9 \pm 5,1$ & & & & & & \\
\hline WAIS-R, "span" de dígitos & $11,9 \pm 3,6$ & $12,2 \pm 3,2$ & 153 & NS & $12,8 \pm 5,2$ & 139 & NS \\
\hline WAIS-R, clave de números & $45,1 \pm 13,8$ & $47,9 \pm 14,3$ & 153 & $<0,0005$ & $49,0 \pm 15,2$ & 139 & 0,012 \\
\hline WAIS-R, bloques & $30,0 \pm 9,7$ & $31,4 \pm 9,8$ & 151 & 0,001 & $33,1 \pm 10,6$ & 139 & 0,002 \\
\hline \multicolumn{8}{|c|}{ Memoria (puntuaciones, media \pm d.e.) } \\
\hline $\begin{array}{l}\text { WMS-memoria lógica, recuerdo } \\
\text { inmediato de historia }\end{array}$ & $9,4 \pm 3,5$ & $10,7 \pm 3,6$ & 153 & $<0,0005$ & $11,3 \pm 3,7$ & 139 & 0,007 \\
\hline $\begin{array}{l}\text { WMS-memoria lógica, recuerdo } \\
\text { diferido de historia }\end{array}$ & $7,6 \pm 3,8$ & $9,1 \pm 3,8$ & 152 & $<0,0005$ & $9,9 \pm 4,0$ & 139 & 0,001 \\
\hline Figura compleja, CFT & $34,8 \pm 2,8$ & $34,4 \pm 2,9$ & 152 & NS & $34,5 \pm 2,5$ & 138 & NS \\
\hline CFT, recuerdo diferido & $16,1 \pm 7,1$ & $19,2 \pm 6,8$ & 152 & $<0,0005$ & $20,8 \pm 7,0$ & 138 & $<0,0005$ \\
\hline
\end{tabular}

Función ejecutiva, flexibilidad, control y velocidad motora (tiempo en segundos, media \pm d.e.)

TMT, diferencia de velocidad de

funcionamiento en partes В у A $\quad 41,3 \pm 31,2 \quad 40,1 \pm 34,7 \quad 152 \quad$ NS $\quad 37,9 \pm 34,8 \quad 138 \quad$ NS

Stroop, diferencia de velocidad de funcionamiento en partes B y A $\quad 27,5 \pm 16,0 \quad 23,7 \pm 13,8 \quad 152<0,0005 \quad 22,3 \pm 12,8 \quad 137 \quad$ NS

Quejas de memoria (puntuaciones, media \pm d.e.)

\begin{tabular}{llllllll}
\hline MCQ & $28,3 \pm 4,1$ & $27,3 \pm 4,7$ & 153 & $<0,0005$ & $27,7 \pm 4,7$ & 139 & NS
\end{tabular}

(1) Entre el comienzo del tratamiento y los 6 meses de seguimiento.

(2) Entre los 6 y los 12 meses de seguimiento. 
Tabla II

Cambios en el estado de ánimo, síntomas mentales y capacidad psicosocial en el seguimiento a los 6 y 12 meses en pacientes adultos deprimidos ambulatorios

\begin{tabular}{|c|c|c|c|c|}
\hline $\begin{array}{c}\mathrm{Al} \\
\text { comienzo } \\
\text { del tratamiento } \\
(\mathrm{n}=174)\end{array}$ & $\begin{array}{c}\text { A los } \\
6 \\
\text { meses } \\
(n=156)\end{array}$ & g.l. & $\mathrm{p}^{1}$ & $\begin{array}{c}\text { A los } \\
6 \\
\text { meses } \\
(n=145)\end{array}$ \\
\hline
\end{tabular}

\begin{tabular}{lrlllrlr}
\hline Depresión (puntuaciones, media \pm d.e.) \\
\hline BDI & $19,9 \pm 9,0$ & $12,7 \pm 9,7$ & 164 & $<0,0005$ & $11,1 \pm 9,4$ & 153 &, 033 \\
HDRS & $17,3 \pm 6,6$ & $11,1 \pm 6,8$ & 168 & $<0,0005$ & $9,1 \pm 6,6$ & $159<0,0005$
\end{tabular}

Síntomas mentales (puntuaciones, media \pm d.e.)

\begin{tabular}{lccccccr}
\hline TAS-20 & $55,1 \pm 11,7$ & $49,4 \pm 12,6$ & 166 & $<0,0005$ & $48,3 \pm 13,0$ & 158 & NS \\
SCL-90, Total & $2,4 \pm 0,6$ & $2,0 \pm 0,6$ & 167 & $<0,0005$ & $1,9 \pm 0,6$ & 159 & 0,029 \\
\hline Capacidad psicosocial (puntuaciones, media \pm d.e.) & & & & & \\
\hline LS & $13,8 \pm 3,3$ & $11,0 \pm 4,0$ & 167 & $<0,0005$ & $10,8 \pm 4,1$ & 158 & NS \\
SOFAS & $62,1 \pm 9,5$ & $65,9 \pm 11,3$ & 166 & $<0,0005$ & $67,9 \pm 11,2$ & 155 & 0,026 \\
\hline
\end{tabular}

(1) Entre el comienzo del tratamiento y los 6 meses de seguimiento.

(2) Entre los 6 y los 12 meses de seguimiento.

en el WMS) (Tabla I). En relación a estas escalas, la mejora fue más aparente durante la primera que en la segunda mitad del seguimiento. No hubo mejoría en memoria verbal inmediata ("Span" de dígitos) o en la flexibilidad, control y velocidad motora (TMT) durante los 12 meses de seguimiento. Hubo una mejoría continua durante el seguimiento en el recuerdo diferido del material visual (CFT), pero no en la memoria inmediata. Se documentó que el uso eficiente de las estrategias de aprendizaje ("List learning") había mejorado sólo durante la segunda mitad del seguimiento. Se encontró una disminución en las quejas de memoria (MCQ) durante la primera, pero no en la segunda mitad del seguimiento.

Los síntomas depresivos (BDI, HDRS) mejoraron durante el seguimiento (Tabla II), pero el cambio fue más marcado en los primeros seis meses. Las puntuaciones medias del TAS-20 disminuyeron de forma significativa durante los primeros seis meses pero no se documentaron cambios entre los seis y doce meses de seguimiento. Se encontró un cambio similar en la media de las puntuaciones sobre satisfacción vital (LS). Los síntomas mentales (SCL-90) mejoraron de manera aparente durante la primera parte del seguimiento y continuaron mejorando, en menor grado, durante la segunda parte. Se documentó una mejoría en el funcionamiento social y ocupacional (SOFAS) durante todo el seguimiento.

El cambio en las quejas de memoria (cambio MCQ) durante los doce meses de seguimiento no se asoció con cambios en el funcionamiento cognoscitivo (Tabla III), mientras que se encontró una asociación significativa entre el cambio MCQ y los cambios en el estado de ánimo (BDI, HDRS), características alexitímicas (TAS20), síntomas mentales (SCL-90), y capacidad psicosocial (SOFAS).

El modelo final en los análisis de regresión logística multivariante incluyeron las siguientes variables: edad, sexo, puntuaciones iniciales BDI (BDI < 19 / BDI $\geq 19$ ), MCQ (MCQ < 28 / MCQ $\geq 29$ ) y memoria 
Tabla III

Comparación de los cambios (media \pm d.e.) en la cognición, estado de ánimo y capacidad psicosocial entre pacientes con disminución en sus quejas de memoria y aquéllos en los que aumentaron o permanecieron invariables tras 12 meses de seguimiento.

Cambio en las quejas de memoria durante el seguimiento a 12 meses

\begin{tabular}{|c|c|c|c|c|}
\hline & $\begin{array}{l}\text { Disminución } \\
\text { de quejas } \\
\text { de memoria } \\
(\mathrm{n}=50)\end{array}$ & $\begin{array}{c}\text { Aumento o sin } \\
\text { cambios en quejas } \\
\text { de memoria } \\
(\mathrm{n}=94)\end{array}$ & g.l. & $\mathrm{p}$ \\
\hline \multicolumn{5}{|l|}{ Cambios en la cognición general } \\
\hline WAIS-R, "span” de dígitos & $0,3 \pm 2,3$ & $1,1 \pm 6,0$ & 142 & NS \\
\hline WAIS-R, clave de números & $5,0 \pm 4,7$ & $3,1 \pm 7,9$ & 142 & NS \\
\hline WAIS-R, bloques & $2,5 \pm 4,9$ & $3,2 \pm 6,0$ & 142 & NS \\
\hline \multicolumn{5}{|l|}{ Cambios de memoria } \\
\hline $\begin{array}{l}\text { WMS, memoria lógica, recuerdo } \\
\text { inmediato de historia }\end{array}$ & $2,2 \pm 2,4$ & $1,7 \pm 2,6$ & 142 & NS \\
\hline $\begin{array}{l}\text { WMS, memoria lógica, recuerdo } \\
\text { diferido de historia }\end{array}$ & $2.9 \pm 2.7$ & $2,0 \pm 2,3$ & 141 & NS \\
\hline Figura compleja, CFT & $-0,3 \pm 2,4$ & $-0,2 \pm 2,9$ & 142 & NS \\
\hline CFT, recuerdo diferido & $4,8 \pm 5,0$ & $5,1 \pm 5,2$ & 142 & NS \\
\hline \multicolumn{5}{|c|}{ Función ejecutiva, flexibilidad, control y velocidad motora, cambios } \\
\hline $\begin{array}{l}\text { TMT, diferencia de velocidad de } \\
\text { funcionamiento en partes B y A }\end{array}$ & $-4,0 \pm 24,7$ & $-1,9 \pm 31,2$ & 142 & NS \\
\hline $\begin{array}{l}\text { Stroop, diferencia de velocidad de } \\
\text { funcionamiento en partes B y A }\end{array}$ & $5,9 \pm 10,9$ & $-4,3 \pm 11,5$ & 141 & NS \\
\hline \multicolumn{5}{|c|}{ Síntomas psíquicos depresivos, manejo psicosocial y ocupacional, cambios } \\
\hline BDI & $-11,2 \pm 9,3$ & $-7,2 \pm 10,0$ & 140 & 0,020 \\
\hline HDRS & $-10,4 \pm 6,1$ & $-6,7 \pm 7,6$ & 142 & 0,004 \\
\hline SCL-90, Total & $-0,8 \pm 0,5$ & $-0,5 \pm 0,6$ & 142 & 0,004 \\
\hline Tas-20 & $-11,4 \pm 10,8$ & $-3,7 \pm 10,1$ & 142 & $<0,0005$ \\
\hline LS & $-3,9 \pm 3,5$ & $-2,6 \pm 4,5$ & 141 & NS \\
\hline SOFAS & $9,9 \pm 9,8$ & $3,3 \pm 10,4$ & 140 & $<0,0005$ \\
\hline
\end{tabular}

lógica, cambio en BDI y cambio en aprendizaje de listas durante todo el seguimiento. Sólo los cambios en la puntuación BDI (OR 0,$95 ;$ CI95\% 0,91 - 1,00; p < 0,05) y en aprendizaje de listas (OR 0,92; CI95\% 0,85$0,99 ; \mathrm{p}<0,05)$ se asociaron independientemente con los cambios en las puntuaciones MCQ.

Se utilizaron análisis de varianza para medidas repetidas para estudiar, posteriormente, las posibles relaciones entre tres variables: quejas de memoria, estado de ánimo y funcionamiento cognoscitivo. Las variables incluídas en el modelo fueron las puntuaciones MCQ, aprendizaje de listas y BDI en la evaluación inicial y a los 6 y 12 meses de seguimiento. Las puntuaciones BDI y del aprendizaje de listas en diferentes momentos en el tiempo se utilizaron como covariables cuando fue adecuado. En el análisis, los sujetos estaban divididos en dos grupos: aquéllos que padecían depresión 
moderada o grave en la evaluación inicial (BDI $\geq 19, \mathrm{n}=72$ ) y los otros (BDI < 19, $\mathrm{n}$ $=62$ ). El análisis mostró que los cambios en el MCQ eran similares en estos dos grupos $(\mathrm{F}(2,262)=0,77, \mathrm{p}=0,465)$. Además, se encontraron diferencias significativas $(\mathrm{p}=$ 0,022 ) entre las puntuaciones MCQ en diferentes momentos, incluso cuando las covariables se excluían del análisis. Tanto el aprendizaje de listas como las puntuaciones BDI en diferentes momentos ejercieron un efecto conjunto en los cambios MCQ $(\mathrm{F}(2,262)=11,75, \mathrm{p}<0,0005)$. Los cambios en MCQ entre diferentes momentos no fueron significativos $(\mathrm{F}(2,262)=2,28, \mathrm{p}=$ $0,104)$ si se incluía en el análisis el efecto de los cambios en el aprendizaje de listas y puntuaciones BDI. Por tanto, los cambios en las puntuaciones MCQ entre diferentes momentos se asociaron a cambios del BDI si se analizaban independientemente del aprendizaje de listas.

\section{Discusión}

Las quejas de memoria disminuyeron durante la primera mitad del seguimiento a los doce meses, pero durante la segunda mitad no hubo cambios. La muestra incluyó pacientes cuyas quejas de memoria disminuyeron y otros cuyas quejas aumentaron. Aunque los síntomas depresivos mejoraron durante todo el seguimiento, el cambio fue más significativo durante los primeros seis meses. Como media, el funcionamiento cognoscitivo mejoró de acuerdo con la mayoría de los tests utilizados en nuestro estudio.

El principal hallazgo de nuestro estudio fue que el cambio en las quejas de memoria en los doce meses de seguimiento se asoció con un cambio en el estado de ánimo, en los síntomas mentales, en las características alexitímicas y en la capacidad funcional, pero no en el funcionamiento cognoscitivo. Esto confirma nuestra observación previa de la misma muestra tras seis meses de seguimiento (Antikainen et al. 2001). Sin embargo, en los análisis de regresión logística ambos, el cambio BDI y el cambio en la puntuación del aprendizaje de listas (función de memoria), se asociaron de manera independiente con cambios en las quejas de memoria. Este resultado se ratificó más tarde mediante un análisis de la varianza de medidas repetidas. El análisis mostró que los cambios en el MCQ estaban asociados con cambios del BDI y del aprendizaje de listas. El resultado fue independiente del nivel de depresión (puntuación BDI) al inicio. Por tanto, en nuestro análisis, el cambio en el estado de ánimo se asoció principalmente con quejas de memoria.

En la mayoría de los estudios anteriores, se ha investigado el deterioro subjetivo de memoria en relación con posibles cambios en el SNC, incluyendo varios tipos de demencia, en muestras de población anciana. Se ha documentado que las quejas de memoria están asociadas a cambios en la función cognoscitiva en algunos estudios (Geerlings et al. 1999) y al estado de ánimo y a rasgos de personalidad en otros (Derouesne et al. 1999). Estos hallazgos, de alguna forma contradictorios, fueron el punto de partida de nuestro estudio de seguimiento; nuestros resultados apoyan la última de las dos hipótesis.

A pesar de que los trastornos subjetivos de memoria y las preocupaciones sobre la pérdida de memoria son comunes, los estudios previos se han centrado casi exclusivamente en población geriátrica y pocos han incluído un seguimiento de los sujetos. Los pacientes de nuestra muestra han estado padeciendo un estado de ánimo depresivo 
durante varios años y fueron remitidos para tratamiento de los síntomas depresivos. Por lo tanto, el diseño de nuestro estudio se centró en la relación entre el estado de ánimo y las quejas de memoria en un marco clínico. Además, nuestro estudio monitorizó el funcionamiento de la memoria y de la función cognoscitiva, además del estado de ánimo, rasgos de personalidad y capacidad psicosocial utilizando una batería versátil de tests. Nuestra aproximación fue observacional y los resultados, por tanto, describieron de forma precisa factores asociados con la recuperación de la depresión en adultos sin enfermedades neurológicas. Diecinueve pacientes de nuestra muestra no reunieron los criterios DSM (SCID I) para trastorno depresivo. Sin embargo, las puntuaciones BDI y HDRS indicaron un estado de ánimo depresivo en este grupo. En el seguimiento a los seis meses encontramos que los cambios en las quejas de memoria y la calidad del tratamiento farmacológico recibido durante el seguimiento parecían no estar relacionados (Antikainen et al. 2001).

Repetimos todas las medidas, incluyendo los rendimientos cognoscitivos, tres veces, lo que proporcionó una oportunidad para observar posibles cambios no lineales en las variables. Las mediciones repetidas pueden ser una fuente de error por el efecto aprendizaje, que sólo puede excluirse mediante un grupo de comparación. Sin embargo, en un estudio clínico es difícil diseñar un estudio tan complicado.

Nuestros resultados sugieren que los pacientes adultos deprimidos que se quejan de problemas de memoria podrían tener una razón adicional para buscar tratamiento para sus problemas afectivos, si saben que sus problemas subjetivos de memoria pueden mejorar al mejorar su depresión.

\section{Bibliografía}

ÄIKIÄ, M., KÄLVIÄINEN, R., RIEKKINEN, P.J. Verbal learning and memory in newly diagnosed partial epilepsy. Epilepsy Research, 22, 157-164, 1995.

ANTIKAINEN, R., HÄNNINEN, T., HONKALAMPI, K., HINTIKKA, J., KOIVUMAA-HONKANEN, H., TANSKANEN, A., VIINAMÄKI, H. Mood improvement reduces memory complaints in depressed patients. European Archives of Psychiatry and Clinical Neuroscience, 251, 6-11, 2001.

BAGBY, R.M., PARKER, J.D.A., TAYLOR, G.J. The Twenty-item Toronto Alexithymia Scale-I: item selection and cross-validation of the factor structure. Journal of Psychosomatic Research, 38, 23-32, 1994.

BECK, A.T., STEER, R.A., GARBIN, M.G. Psychometric properties Beck Depression Inventory: Twenty-five years of evaluation. Clinical Psychology Review, 8, 77$100,1988$.

CHANDLER, J.D., GERNDT, J. Memory complaints and memory deficits in young and old psychiatric inpatients. Journal of Geriatric Psychiatry and Neurology, 1, 84-88, 1988.

CLARNETTE, R.M., ALMEIDA, O.P., FORSTI, H., PATON, A., MARTINS, R.N. Clinical characteristics of individuals with subjective memory loss in Western Australia: results from a cross-sectional survey. International Journal of Geriatric Psychiatry, 16, 168-174, 2001.

COMIJS, H., DEEG, D., DIK, M., TWISK, J., JONKER, C. Memory complaints; the association with psycho-affective and health problems and the role of personality characteristics. A 6-year follow-up study. Journal of Affective Disorders, 72, 157-165, 2002.

COMMISSARIS, C.J., PONDS, R.W., JOLLES, J. Subjective forgetfulness in a normal Dutch population: possibilities for health education and other interventions. Patient Education and Counseling, 34, 25-32, 1998.

CROOK, T.H., FEHER, E.P., LARRABEE, G.J. Assessment of memory complaint in age-associated memory impairment: The MAC-Q. International Psychogeriatrics, 4, 165-176, 1992.

DEROGATIS, L.R., LIPMAN, R.S., COVI, L. The SCL-90: an outpatient psychiatric rating scale - preliminary report. Psychopharmacology Bulletin, 9, 13-28, 1973.

DEROUESNE, C., LACOMBLEZ, L. La plainte mnésique: épidémiologie et démarche diagnostique. Presse Medicale, 29, 858-862, 2000. 
DEROUESNE, C., LACOMBLEZ, L., THIBAULT, S., LEPONCIN, M. Memory complaints in young and elderly subjects. International Journal of Geriatric Psychiatry, 14, 291-301, 1999.

GEERLINGS, M.I., JONKER, C., BOUTER, L.M., ADER, H.J., SCHMAND, B. Association between memory complaints and incident Alzheimer's disease in elderly people with normal baseline cognition. American Journal of Psychiatry, 156, 531-537, 1999.

GOLDEN, C.J. Stroop Color and Word Test. Chicago: Stoelting Company, 1978.

GOLDMAN, H.H., SKODOL, A.E., LAVE, T.R. Revising Axis V for DSM-IV: A review of measures of social functioning. American Journal of Psychiatry, 149, 1148$1156,1992$.

HAMILTON, M.A. A rating scale for depression. The Journal of Neurology, Neurosurgery, and Psychiatry, 23, 56-62, 1960.

HOLI, M., SAMMALlAHTI, P., AALBERG, V. A Finnish validation study of the SCL-90. Acta Psychiatrica Scandinavica, 97, 42-46, 1998.

JONKER, C., GEERLINGS, M.I., SCHMAND, B. Are memory complaints predictive for dementia? A review of clinical and population-based studies. International Journal of Geriatric Psychiatry, 15, 983-991, 2000.

JORM, A.F., CHRISTENSEN, H., KORTEN, A.E., JACOMB, P.A., HENDERSON, S.A. Memory complaints as a precursor of memory impairment in older people: a longitudinal analysis over 7-8 years. Psychological Medicine, 31, 441-449, 2001.

JOUKAMAA, M., MIETTUNEN, J., KOKKONEN, P., KOSKINEN, M., JULKUNEN, J., KAUHANEN, J., JOKELAINEN, J., VEIJOLA, J., LÄKSY, K., JÄRVELIN, M-R. Psychometric properties of the Finnish 20-item Toronto Alexithymia Scale. Nordic Journal of Psychiatry, 55, 123-127, 2001.

KOIVUMAA-HONKANEN, H.T., HONKANEN, R., ANTIKAINEN, R., HINTIKKA, J., VIINAMÄKI, H. Self reported life satisfaction and treatment factors in patients with schizophrenia, major depression and anxiety disorder. Acta Psychiatrica Scandinavica, 99, 377-382, 1999.

LEZAK, M.D. Neuropsychological assessment. $3^{\text {rd }}$ ed. New York: Oxford University Press, 325-326, 1999.
McDOnAld, E., COPE, H., DAVID, A. Cognitive impairment in patients with chronic fatigue: a preliminary study. Journal of Neurology, Neurosurgery and Psychiatry, 56, 812-815, 1993.

REISCHIES, F.M., NEU, P. Comorbidity of mild cognitive disorder and depression. A neuropsychological analysis. European Archives of Psychiatry and Clinical Neuroscience, 250, 186-193, 2000.

REITAN, R.M. Validity of the Trail Making Test as an indicator of organic brain damage. Perceptual and Motor Skills, 8, 271-276, 1958.

ROHLING, M.L., GREEN, P., ALLEN, L.M., IVERSON, G.L. Depressive symptoms and neurocognitive test scores in patients passing symptom validity test. Archives of Clinical Neuropsychology, 17, 205-222, 2002.

SPITZER, R., WILLIAMS, J.B.W., GIBBON, M., FIRST, M.B. The structure of interview for DSM III-R (SCID) I: History, rationale and description. Archives of General Psychiatry, 49, 624-629, 1992.

SPITZER, R., WILLIAMS, J., GIBBON, M., FIRST, M. Users guide for the Structured Clinical Interview for DSMIII-R. Washington: American Psychiatric Press, 1990.

STEWART, R., RUSS, C., RICHARDS, M., BRAYNE, C., LOVESTONE, S., MANN, A. Depression, APOE genotype and subjective memory impairment: a cross-sectional study in an African-Caribbean population. Psychological Medicine, 31, 431-440, 2001.

WECHSLER, D. Wechsler memory scale manual. San Antonio: The Psychological Corporation, 1974.

WECHSLER, D. Wechsler adult intelligence scale Revised. Cleveland: The Psychological Corporation, 1981.

WHO. The international statistical classification of diseases, injuries, and causes of death, tenth revision, vol. 1 . Tabular list. Geneva: World Health Organization, 1992.

Dirección para correspondencia:

Risto Antikainen, Ph.D.

Kuopio University Hospital

Department of Psychiatry

P.O. Box 1777

FIN-70211 Kuopio

FINLAND

e-mail: risto.antikainen@kuh.fi 